\title{
Empagliflozin Alleviates Diabetic Renal Tubular Lipid Accumulation and NLRP3 Inflammasome Activation Through AGEs-RAGE Pathway
}

\section{Zheng Liu}

Soochow University Affiliated No 1 Peoplel's Hospital: First Affiliated Hospital of Soochow University Juan Chen

Jiangsu Provincial Institute of Traditional Chinese Medicine: Jiangsu Province Academy of Traditional Chinese Medicine

\section{Lili Sun}

Soochow University Affiliated No 1 Peoplel's Hospital: First Affiliated Hospital of Soochow University

Jianzhong Li

Soochow University Affiliated No 1 Peoplel's Hospital: First Affiliated Hospital of Soochow University Hong Sun ( $\nabla$ sunhong_611@126.com )

First Affiliated Hospital of Soochow University https://orcid.org/0000-0003-4335-2789

\section{Research}

Keywords: diabetic kidney disease, advanced glycation end products, empagliflozin, sterol regulatory element binding protein cleavage-activating protein, endoplasmic reticulum stress

Posted Date: September 13th, 2021

DOl: https://doi.org/10.21203/rs.3.rs-870754/v1

License: @ (i) This work is licensed under a Creative Commons Attribution 4.0 International License. Read Full License 
1 Empagliflozin alleviates diabetic renal tubular lipid accumulation and NLRP3

2 inflammasome activation through AGEs-RAGE pathway

$4{ }^{1}$ Department of Endocrinology and Metabolism, The First Affiliated Hospital of

5 Soochow University, Suzhou, Jiangsu, China

$6 \quad{ }^{2}$ Department of Endocrinology, Jiangsu Province Hospital of Chinese Medicine,

7 Affiliated Hospital of Nanjing University of Chinese Medicine, Nanjing, China

$8{ }^{3}$ Department of Nephrology, The First Affiliated Hospital of Soochow University,

9 Suzhou, Jiangsu, China

10

*Correspondence to: Hong Sun E-mail: sunhong_611@126.com

Li Jianzhong E-mail:ljzsnk@ hotmail.com

$\$$ These authors contributed equally to this work. 


\begin{abstract}
:
Background: Advanced glycation end products (AGEs) are pathogenic factors of renal tubular lipid accumulation and play a negative role in diabetic kidney disease (DKD). Glucose cotransporter (SGLT) 2 inhibition offers strong renoprotection in the progression of DKD. The aim of the current study was to investigate the effects of empagliflozin (EMPA, a potent and selective SGLT2 inhibitor) on AGEs-induced renal tubular lipid accumulation in both diabetic mice fed with a high-AGEs diet and AGEs-treated cultured human renal proximal tubular epithelial (HK-2) cells.

Methods: In vivo, EMPA was used to treat $\mathrm{db} / \mathrm{db}$ mice fed a high-AGEs diet or an AIN-76 basal diet. In an in vitro study, HK-2 cells were treated with AGEs-bovine serum albumin (BSA) and/or EMPA. Sterol regulatory element binding protein (SREBP) cleavage-activating protein (SCAP) translocation was detected by confocal microscopy.
\end{abstract}

Results: EMPA reduced tubular lipid droplets and intracellular cholesterol content, as well as the expression of proteins involved in the synthesis and absorption of cholesterol in the kidneys of basal diet-fed db/db mice, high-AGEs diet-fed db/db mice and AGEs-BSA-treated HK-2 cells. AGEs-BSA loading promoted the formation of SCAP-SREBP-2 complexes and enhanced the transport of the complexes to the Golgi, but these effects were markedly inhibited by EMPA in HK-2 cells. EMPA reduced renal inflammation both in basal diet-fed $\mathrm{db} / \mathrm{db}$ mice and high-AGEs diet-fed $\mathrm{db} / \mathrm{db}$ mice, and suppressed NLRP3 inflammasome activation in AGEs-BSA-treated HK-2 cells. In addition, EMPA reduced the serum AGEs level in vivo and inhibited renal tubular endoplasmic reticulum (ER) stress and receptor of AGEs (RAGE) expression both in vivo and in vitro.

Conclusions: EMPA attenuated AGEs synthesis and inhibited the AGEs-RAGE signaling pathway, thereby suppressing ER stress and inhibiting abnormal cholesterol metabolism and release of inflammatory cytokines, thus alleviating renal tubular lipid accumulation and inflammation.

Keywords: diabetic kidney disease, advanced glycation end products, empagliflozin, sterol regulatory element binding protein cleavage-activating protein, endoplasmic reticulum stress 


\section{Background}

Diabetic kidney disease (DKD) is a chronic kidney disease caused by diabetes, and almost $40 \%$ of diabetic patients with DKD [1]. DKD plays a major role in the global burden of disease. Although the research on DKD has lasted for many years, the comprehensive mechanism of DKD is still not fully understood. Metabolic changes associated with diabetes lead to renal pathological changes and functional damage, which are generally considered the leading causes of DKD. Among the many key metabolic changes that lead to the onset of DKD, nonenzymatic glycosylation (NEG) is a significant presence. NEG attaches reducing sugar to the free amino group of protein through a series of events, which is irreversible. In this process, Schiff base and Amadori products are formed, and advanced glycation end products (AGEs) are finally produced [2]. In addition, the serum level of AGEs in patients with diabetes complicated with renal insufficiency will be significantly increased in this process. What's more, renal structure will suffer progressive damage caused by renal AGEs, which will further lead to renal function damage in DKD patients [3].

Through the integration and analysis of many years of research, it can be found that some previous description of the characteristic histological changes of DKD is not comprehensive, and the focus is on diffuse and nodular glomerulosclerosis. However, in the aspect of renal insufficiency of DKD, changes within the tubulointerstitium are more important than glomerulopathy $[4,5]$. Some studies have shown that AGEs play important roles in tubular injury during DKD. Excessive levels of AGEs can cause renal tubular oxidative stress [6], inflammatory responses [7], and even apoptosis [8]. In our previous study, we have reported that AGEs could induce low-density lipoprotein receptor (LDLr)-mediated cholesterol uptake and 3-hydroxy-3-methylglutaryl coenzyme A reductase (HMGCoAR)-mediated cholesterol synthesis. After the successful uptake and synthesis of cholesterol, a foam cell can be formed in the human renal tubular epithelial cell line (HK-2). [9, 10]. LDLr and HMGCoAR are mainly regulated by sterol regulatory element binding protein-2 (SREBP-2) [11]. SREBP cleavage-activating protein (SCAP) is the chaperone of SREBP-2 [12]. When cells have an obvious demand for cholesterol, SCAP will transports SREBP-2 from the endoplasmic reticulum (ER) to the Golgi and activates it through proteolytic cleavage. The N-terminal fragment of SREBP-2 (nSREBP-2) is transferred into the nucleus after cutting and processing. In the nucleus, nSREBP-2 actively activates LDLr and HMGCoAR, which will increase the uptake and synthesis of cholesterol. However, when there is no obvious demand for cholesterol in cells, the SCAP-SREBP-2 complexes will be retained in the ER, thus down regulating the expression of LDLR and HMGCoAR. This kind of feedback regulation mediated by SCAP plays an indispensable role in preventing the overload of intracellular cholesterol content under physiological conditions [13]. However, it may be forcibly destroyed in the state of disease, such as type 2 diabetes mellitus (T2DM) [14].

In recent years, with the development of science and technology, antidiabetic drugs have also been updated. Nowadays, the newly developed glucose cotransporter 
(SGLT) 2 inhibitor has attracted increasingly attention due to its unique therapeutic principle and better therapeutic effect. SGLT2 is located on the apical side of proximal tubular cells, accounting for the majority of renal glucose reabsorption, as high as $90 \%$ [15]. The therapeutic principle of SGLT2 inhibitor is to reduce the blood glucose level by increasing the excretion of glucose. Evidence from randomized clinical trials (RCTs) has shown an unexpected benefit and safety of these inhibitors in renal outcomes irrespective of their impact on glycemic control [16]. SGLT2 inhibitors can directly mitigate kidney damage by suppressing numerous pathways linked to tubular hypoxia and fibrosis, such as oxidative stress and inflammation [17-19]. What's more, Wang XX et al. reported that an SGLT2 inhibitor could modulate renal lipid metabolism and prevent the development of DKD in $\mathrm{db} / \mathrm{db}$ mice [20]. However, the specific mechanism remains unclear. Hence, the current study was undertaken to explore the mechanism of empagliflozin (EMPA), a potent and selective SGLT2 inhibitor, in improving renal lipid deposition in a T2DM mouse model fed with a high-AGEs diet and AGEs-treated HK-2 cells.

\section{Methods}

2.1 Animal experimental design

The male $\mathrm{db} / \mathrm{m}$ mice and $\mathrm{db} / \mathrm{db}$ mice were obtained from the National Model Animal Centre of Nanjing University (Nanjing, China) and all of them had C57BL/KsJ gene background. This study follows the latest edition of Helsinki Manifesto and adopts the protocol approved by the Ethics Committee of Soochow University. The mice were placed in polypropylene cages and the room temperature was controlled at a certain standard $\left(22 \pm 2^{\circ} \mathrm{C}\right)$, the humidity was kept within $60 \pm 5 \%$, and the light was controlled at 12:12 hour light:dark cycle. After 2 weeks of adaptation, some of the 8-week-old $\mathrm{db} / \mathrm{db}$ mice were fed an AIN-76 basal diet (Xietong Biology Co., Ltd., Nanjing, China), and other mice were fed a high-AGEs diet. The high-AGEs diet is based on AIN-76 basic diet. After heat exposure $\left(90^{\circ} \mathrm{C}\right.$ for $10 \mathrm{~min}$ ), AIN-76 basic diet will generate diet-derived AGEs [21]. Then, the mice were divided into five groups consisting of five animals each as follows: Group 1, db/m mice fed an AIN-76 basal diet (db/m) served as a control; Group 2, $\mathrm{db} / \mathrm{db}$ mice fed an AIN-76 basal diet (db/db); Group 3, db/db mice fed an AIN-76 basal diet and administered $10 \mathrm{mg} / \mathrm{kg}$ EMPA (MedChemExpress, China) by oral gavage once daily from the sixth week and lasting for two weeks (db/db+EMPA); Group 4, db/db mice fed a high-AGEs diet (db/db+AGEs); Group 5, db/db mice fed a high-AGEs diet and administered EMPA as in Group 3 $(\mathrm{db} / \mathrm{db}+\mathrm{AGEs}+\mathrm{EMPA})$. A single mouse was placed in a special metabolic cage to collect urine excreted within 24 hours. At the end of the 8th week, that is, the last week of the experiment, we began to collect samples for follow-up studies and collate relevant data. The blood samples were used for subsequent biological and chemical analysis. In addition, kidney samples were used for histological evaluation later.

\subsection{Biochemical assays}


After the experimental period, the mice were sacrificed, blood samples were collected from the right ventricle, which was used for subsequent biochemical analysis. The concentrations of fasting blood glucose (FBG), total triglycerides (TG), total cholesterol (TC), blood urea nitrogen (BUN) and serum creatinine (SCR) were determined using a fully automatic biochemical analyzer (Hitachi). Using ELISA provided by CUSABIO (Wuhan, China) to measure the serum AGEs and urinary neutrophil gelatinase-associated lipocalin (u-NGAL, a marker of renal tubular injury), and 24-hour urine protein was measured by Coomassie brilliant blue protein assay (Jiancheng Bioengineering Institute, Nanjing, Jiangsu).

2.3 Renal morphology

The paraffin embedded tissues were sectioned from the renal cortex, collected completely and preserved reasonably. Gelatin coated slides were selected and cross-sections $(3 \mu \mathrm{m})$ of previously collected slides were placed on them and stained with hematoxylin-eosin (HE) staining.

\subsection{Cell culture}

HK-2 cells which the source of culture is the American Type Culture Collection (Manassas, VA, USA) were cultured in RPMI 1640 medium (HyClone; Logan, UT, USA) in a cell culture incubator under $37^{\circ} \mathrm{C}$ with $95 \%$ air and $5 \% \mathrm{CO} 2$ for $24 \mathrm{~h}$. This medium contained 10\% fetal calf serum (HyClone; GE Healthcare Life Sciences, Logan, UT, USA), 2 mM L-glutamine solution, $100 \mathrm{U} / \mathrm{ml}$ penicillin and $100 \mu \mathrm{g} / \mathrm{ml}$ streptomycin (Sigma-Aldrich; Merck KGaA). All experiments were performed in serum-free RPMI 1640 medium, which contained $0.2 \%$ bovine serum albumin (BSA; Sigma-Aldrich; Merck KGaA). AGEs-BSA was purchased from Abcam (Cambridge, UK), while EMPA was purchased from MedChemExpress (Shanghai, China). HK-2 cells were placed in the experimental medium, which contained $200 \mu \mathrm{g} / \mathrm{ml}$ AGEs-BSA, $500 \mathrm{nM}$ EMPA, or $200 \mu \mathrm{g} / \mathrm{ml}$ AGEs-BSA plus 500 nM EMPA for 48 hours.

\subsection{Observation of lipid accumulation}

The lipid accumulation in HK-2 cells and kidneys of $\mathrm{db} / \mathrm{db}$ mice was observed by Oil Red O staining. Briefly, there are four key steps. The first is to fix the sample with $4 \%$ paraformaldehyde, the second step is to dye with Oil Red O for 30 minutes, the third step is to dye with hematoxylin for 5 minutes, and the fourth step is to examine the results obtained through the experiment with an optical microscope (Carl Zeiss, Hertfordshire, UK).

\subsection{Quantitative measurement of intracellular cholesterol}

A commonly used method to quantitative measure TC and free cholesterol (FC) in vitro and in vivo is enzymatic analysis (Applygen Technologies Inc., Beijing, China). The formula for calculating the concentration of cholesterol ester (CE) is calculated as TC minus FC. 
2.7 Protein extraction and Western blot analysis

Proteins from whole-cell and nuclear extracts were denatured and then subjected to electrophoresis on $6 \%$ 15\% SDS polyacrylamide gels, which was performed as previously described [22]. After the denatured proteins had been electrophoresed, the polyvinylidene fluoride membranes (GE Healthcare, Buckinghamshire, UK) were chosen to carry out the transfer. After transfer, the blots were placed at room temperature and blocked for 1 hour with $5 \%$ bovine serum albumin in Tris-buffered saline containing 0.05\% Tween 20 (TBST). Then, the blots were washed. After cleaning, the blots were cultured in TBST containing $5 \%$ bovine serum albumin overnight at $4^{\circ} \mathrm{C}$ with a 1:200-1000 dilution of NLRP3, IL-1 $\beta$, SCAP, SREBP-2, LDLr, HMGCoAR, glucose-regulated protein 78 (GRP78), C/EBP-homologous protein (CHOP), receptor of AGEs (RAGE) antibodies and a GAPDH antibody (Abcam, Cambridge, UK). The membranes were washed three times with TBST, incubated with a secondary antibody (1:5000 dilution in TBST containing 1\% bovine serum albumin; Santa Cruz Biotechnology) for $1 \mathrm{~h}$ at room temperature and then washed three times with TBST. After the chemiluminescence reaction (Pierce, Rockford, IL, USA), bands were detected by exposing the blots to X-ray films for the appropriate period. For quantitative analysis, LabWorks software (UVP Laboratory Products, Upland, CA, USA) was used to detect and evaluate the density of the bands, and $\beta$-actin density was used for normalization.

\subsection{Confocal microscopy}

A green fluorescent protein (GFP)-SCAP expression construct was made by ligating human SCAP cDNA into the BstE-XbaI sites of the pEGFP-C1 vector (Genechem Co. Ltd., Shanghai, China), which was performed as previously described[9]. Cells were transfected with pEGFP-SCAP using Effectene Transfection Reagent (Invitrogen, Paisley, UK) according to the manufacturer's protocol. Next, HK-2 cells were plated on chamber slides. After 48 hours of treatment under different experimental conditions, the transfected cells were fixed in $5 \%$ formalin solution for 30 minutes, then infiltrated with $0.25 \%$ Triton X-100 for 15 minutes, and stained with Golgin-97 antibody (Molecular Probes, Inc., Eugene, OR, USA) for $2 \mathrm{~h}$ at room temperature. After washing, the samples were further stained with a secondary fluorescent antibody for 1 hour. Then, a Zeiss LSM 510 Meta (Carl Zeiss, Hertfordshire, UK) was selected for confocal microscopy.

\subsection{Coimmunoprecipitation (Co-IP)}

The activation of the NLRP3 inflammasome and the interaction between SCAP and SREBP-2 in HK-2 cells were analyzed by co-IP. In short, the total protein was extracted in cell IP lysis buffer (Thermo Scientific Pierce, USA). Cell lysates were cleared by centrifugation, and supernatants were immunoprecipitated with the appropriate antibodies using protein A/G-agarose beads. Samples were then subjected to immunoblotting analysis with the antibodies shown. 
2.10 Statistics

SPSS software 20.0 was used to analyze the experimental data. The data were expressed by mean \pm S.E.M. method, and multiple comparisons were made by one-way ANOVA. After the comparison, Bonferroni multiple comparison test was operated. Differences were considered significant when $P$ values $<0.05$.

\section{Results}

3.1 Effects of EMPA on biochemical characteristics of diabetic mice.

At the end of the experiment, the general characteristics of mice were obtained, as shown in Fig. 1. All diabetic mice displayed increased FBG (Fig. 1A), and the levels of AGEs (Fig. 1B), TG (Fig. 1C), TC (Fig. 1D), SCR (Fig. 1E), BUN (Fig. 1F), u-NGAL (Fig. 1G) and 24-hour urine protein (Fig. 1H) in $\mathrm{db} / \mathrm{db}$ mice were higher than those in $\mathrm{db} / \mathrm{m}$ mice. Compared with those in $\mathrm{db} / \mathrm{db}$ mice, the levels of the above parameters in addition to TG, TC and BUN in $\mathrm{db} / \mathrm{db}$ mice fed a high-AGEs diet were significantly elevated. However, EMPA treatment resulted in a remarkable decrease in the levels of FBG, AGEs, SCR, BUN, u-NGAL and 24-hour urine protein while TG and TC showed no change in either basal diet-fed $\mathrm{db} / \mathrm{db}$ mice or $\mathrm{db} / \mathrm{db}$ mice fed a high-AGEs diet.

\subsection{Effects of EMPA on renal lipid accumulation in vivo and in vitro.}

To assess whether EMPA could alleviate lipid accumulation in renal tubular cells, we observed lipid droplets and analyzed the intracellular cholesterol content in the kidneys of $\mathrm{db} / \mathrm{db}$ mice. Oil Red $\mathrm{O}$ staining revealed that the kidney of the $\mathrm{db} / \mathrm{m}$ mice had almost no staining, indicating that there was no lipid deposition in the renal tubules of this group of mice. However, strong positive areas in the renal tubules of $\mathrm{db} / \mathrm{db}$ mice were observed (Fig. 2A). By quantitative analysis of intracellular cholesterol, we obtained a proof of increased cholesterol content in the kidney of $\mathrm{db} / \mathrm{db}$ mice (Fig. 2B). In addition, when $\mathrm{db} / \mathrm{db}$ mice were used as the control group, Oil Red O staining was more obvious and intracellular cholesterol content was more prominent in $\mathrm{db} / \mathrm{db}$ mice fed with a high-AGEs diet (Fig. 1A and B). However, EMPA treatment reduced tubular lipid droplets and the intracellular cholesterol content in both the basal diet-fed $\mathrm{db} / \mathrm{db}$ mice and high-AGEs diet-fed $\mathrm{db} / \mathrm{db}$ mice (Fig. 2A and B).

Next, we investigated the effects of EMPA on lipid accumulation in tubular cells in vitro. As shown in Fig. 2C, compared with the control group, the accumulation of lipid droplets in HK-2 cells treated with AGEs-BSA showed an obvious upward trend. This could be demonstrated by quantitative cholesterol measurements (Fig. 2D). However, EMPA treatment lowered the lipid droplet and intracellular cholesterol content in AGEs-BSA-treated HK-2 cells (Fig. 2C and D).

3.3 Effects of EMPA on renal cholesterol metabolism in vivo and in vitro.

To further study the effect of EMPA on cholesterol metabolism, we analyzed the expression of HMGCoAR, LDLr, SREBP-2, nSREBP-2 and SCAP in the 
kidneys of diabetic mice. As shown in Fig. 3A, the protein levels of HMGCoAR, LDLr, nSREBP-2, SREBP-2 and SCAP in the kidneys of $\mathrm{db} / \mathrm{db}$ mice increased with $\mathrm{db} / \mathrm{m}$ mice as the control group. Taking $\mathrm{db} / \mathrm{db}$ mice as control group, the protein levels of HMGCoAR, LDLr, nSREBP-2, SREBP-2 and SCAP in the kidneys of $\mathrm{db} / \mathrm{db}$ mice fed with a high-AGEs diet were significantly increased. However, after EMPA treatment, the expression of these proteins decreased significantly in the basal diet-fed $\mathrm{db} / \mathrm{db}$ mice, as well as in $\mathrm{db} / \mathrm{db}$ mice fed with a high-AGEs diet.

Next, we examined the effects of EMPA on HMGCoAR, LDLr, SREBP-2, nSREBP-2 and SCAP expression in HK-2 cells. As shown in Fig. 3B, the protein expression of HMGCoAR, LDLr, SREBP-2, nSREBP-2 and SCAP in AGEs-BSA-treated HK-2 cells, which was significantly higher than that in the control, could be suppressed by EMPA treatment in HK-2 cells.

Furthermore, confocal microscopy was used to observe the translocation of SCAP between the endoplasmic reticulum (ER) and the Golgi in HK-2 cells. We found that AGEs-BSA loading played a positive role in the accumulation of SCAP in the Golgi. However, the accumulation of SCAP in HK-2 cells was significantly decreased by EMPA treatment (Fig. 4A). In addition, the result of co-IP showed that the number of SCAP-SREBP-2 complexes was significantly higher in AGEs-BSA-treated HK-2 cells, while EMPA weakened the interaction between SCAP and SREBP-2. These above results suggested that AGEs facilitated the transport of SCAP-SREBP-2 complexes from the ER to the Golgi by upregulating the expression of SCAP and SREBP-2. Thus, the hydrolysis of SREBP-2 was accelerated. Then, nSREBP-2 fragments that entered the nucleus increased in number and bound to the promoters of HMGCoAR and LDLr, which enhanced the transcription and translation of HMGCoAR and LDLr, and caused increased cholesterol synthesis and uptake. However, EMPA dowenregulated the expression of SCAP and SREBP-2, which attenuated the transport of SCAP-SREBP-2 protein complexes, followed by the downregulation of HMGCoAR and LDLr, and ultimately, reduced the synthesis and uptake of cholesterol.

3.4 Effects of EMPA on renal tubular inflammation in diabetic mice and HK-2 cells.

Through the analysis of HE staining, it could be seen that no obvious pathological changes in glomeruli and tubules in $\mathrm{db} / \mathrm{m}$ mice; however, in the kidneys of $\mathrm{db} / \mathrm{db}$ mice, it was obvious that inflammatory cells were infiltrated in the renal interstitium. This change was more prominent in $\mathrm{db} / \mathrm{db}$ mice fed with a high-AGEs diet. However, EMPA improved renal inflammation in basal diet-fed $\mathrm{db} / \mathrm{db}$ mice and high-AGEs diet-fed db/db mice (Fig. 5A).

Next, we analyzed the effect of EMPA on the activation of the NLRP3 inflammasome since the NLRP3 inflammasome is one of the most prominent signaling pathways involved in the pathogenesis of DKD [23]. NLRP3 
inflammasome is composed of three parts: the NOD-like receptor protein 3, the adapter ASC, and pro-caspase-1. These three parts combine to form a platform called intracellular multimeric protein danger-sensing platform. It promotes the autocatalytic activation of pro-caspase- 1 and mediates the proteolytic activation of proinflammatory cytokines, including pro-interleukin (IL)-1 $\beta$. As shown in Fig. 5B, with $\mathrm{db} / \mathrm{m}$ mice as the control group, the protein level of NLRP3 and IL-1 $\beta$ in the kidney of $\mathrm{db} / \mathrm{db}$ mice increased significantly. And these data were higher in the kidneys of $\mathrm{db} / \mathrm{db}$ mice fed a high-AGEs diet. However, the protein expression in $\mathrm{db} / \mathrm{db}$ mice was significantly reduced by EMPA treatment, and the protein expression in $\mathrm{db} / \mathrm{db}$ mice fed with a high-AGEs diet was also affected to a great extent; consistent results were confirmed in HK-2 cells (Fig. 5C). After that, we detected the activation of NLRP3 inflammasome by co-IP in HK-2 cells. The results showed that the number of NLRP3-ASC-caspase-1 protein complexes was significantly higher in AGEs-BSA-treated HK-2 cells, whereas the protein complexes barely formed in EMPA-treated HK-2 cells (Fig. 5D), which indicated that EMPA had a positive effect on the activation of NLRP3 inflammasome, and could inhibit the cleavage and release of IL-1 $\beta$ in renal tubular cells.

\subsection{Effects of EMPA on ER stress and RAGE expression.}

As known that intracellular cholesterol metabolism and NLRP3 inflammasome activation are closely related to ER stress. For further study, we evaluated the expression of GRP-78 (an ER chaperone, the expression of which is increased upon exposure to ER stress) and CHOP (a transcription factor that is activated during excessive ER stress) both in vivo and in vitro. Elevated protein expression levels of GRP-78 and CHOP were observed in the kidneys of the basal diet-fed $\mathrm{db} / \mathrm{db}$ mice and high-AGEs diet-fed $\mathrm{db} / \mathrm{db}$ mice (Fig. 6A), as well as in AGEs-BAS-treated HK-2 cells (Fig. 6B), which indicated that ER stress was triggered by AGEs in the kidneys of diabetic mice and HK-2 cells. However, EMPA treatment inhibited the expression of GRP-78 and CHOP (Fig. 6A and B), suggesting that EMPA could suppress the ER stress induced by AGEs.

There are specific AGEs receptors (RAGE) on the surface of renal tubules. AGEs can bind to RAGE. After the completion of the binding step, ER stress will be activated and specific biological effects will be produced in the progress of DKD [24, 25]. Therefore, we evaluated the expression of RAGE both in vivo and in vitro. The results showed that the protein expression of RAGE was increased in the kidneys of $\mathrm{db} / \mathrm{db}$ mice compared with those of $\mathrm{db} / \mathrm{m}$ mice and was even higher in the kidneys of $\mathrm{db} / \mathrm{db}$ mice fed with a high-AGEs diet (Fig. 6C); at the same time, we also obtained consistent results in HK-2 cells, which further confirmed the above phenomenon (Fig. 6D). However, EMPA treatment clearly reduced the expression of RAGE both in the basal diet-fed $\mathrm{db} / \mathrm{db}$ mice and high-AGEs diet-fed $\mathrm{db} / \mathrm{db}$ mice, and consistent results were confirmed in HK-2 cells (Fig. 6C and D).

\section{Discussion}


This research is groundbreaking to some extent, mainly because this research has created a new research topic, that is, the effect of EMPA on lipid accumulation and inflammation induced by AGEs in diabetic renal tubules. Previously, our team found that renal tubular injury is associated with lipid accumulation in high fat/sucrose diet and streptozotocin-induced T2DM rats [14]. Other studies have shown lipid droplet deposition in the renal tubules of T2DM db/db mice [26], and a similar phenomenon was found in the kidney biopsies of humans with T2DM [27]. However, in this study, we further discovered that increased level of AGEs was a risk factor for renal tubular lipid deposition in $\mathrm{db} / \mathrm{db}$ mice, while EMPA treatment alleviated AGE-induced lipid accumulation both in vivo and in vitro. Although increasing attention has been given to the disorder of renal lipid metabolism in T2DM, most studies have focused on renal triglyceride metabolism, and little attention has been given to the abnormal metabolism of cholesterol. The current study mainly focuses on the disturbed cholesterol feedback regulation, and we found that AGEs upregulated the expression of SCAP and SREBP-2, causing increased formation of SCAP-SREBP-2 complexes. Next, the enhanced transport of the complexes from ER-to-Golgi promoted the hydrolysis of SREBP-2, and then its active fragment (nSREBP-2) entered the nucleus, which accelerated the transcription and translation of HMGCoAR and LDLr, followed by increased cholesterol uptake and synthesis in renal tubular epithelial cells, and ultimately, leading to tubular foam cell formation. However, EMPA could downregulate AGEs induced SCAP and SREBP-2 expression, inhibiting SCAP-SREBP-2 translocation from the ER to the Golgi; thus, the transcription of SREBP-2 target genes declined, resulting in a reduction in cholesterol synthesis and uptake in renal tubules. Although EMPA decreased the content of cholesterol in the kidneys, it did not affect the TC in the blood, which might be related to the change of blood volume and the increased oxidation or intestinal excretion of cholesterol.

Lipid deposition is always accompanied by inflammatory responses in DKD. It has been reported that renal inflammation aggravates the disorder of renal lipid metabolism [28], whereas lipid accumulation stimulates inflammatory responses in the kidneys [29]. Hence, we further studied renal inflammation and the effects of EMPA on it. To make a more comprehensive and detailed exposition of the molecular mechanism of the beneficial effect of EMPA, we decided to focus on a relatively observation unit, namely NLRP3 inflammasome. This is a protein complex that can be found in the development of DKD [23, 30]. A number of studies have reported that AGEs can activate the NLRP3 inflammasome in macrophages, glomerular podocytes and some other cell types [31-33]. However, for the first time, this study demonstrated that AGEs could activate the NLRP3 inflammasome in renal tubular epithelial cells, thus causing the release of IL-1 $\beta$ from renal tubules. Recent studies showed that SCAP-SREBP-2 complexes ER-to-Golgi translocation facilitates the optimal activation of the NLRP3 inflammasome in macrophages [34]. Therefore, we speculated that the transport of the SCAP-SREBP-2 complexes induced by AGEs might contribute to the activation of the NLRP3 inflammasome in renal tubules. In addition, based on previous studies, we know that for those present in C57BL/6 mice, EMPA can inhibit the activation of the 
renal NLRP3 inflammasome induced by a high fat/sugar diet [35]. Here, we confirmed that EMPA decreased AGEs-induced tubular NLRP3 activation. The last but also important point of this effect was that the production of IL-1 $\beta$ in the kidneys of diabetic mice and in HK-2 cells decreased significantly.

The lack of effects on cholesterol metabolism and inflammatory responses in control renal tubular cells suggested that EMPA may not exert a direct role on cholesterol regulation or NLRP3 inflammasome activation. Therefore, ER stress was further evaluated since it is a trigger of disturbed cholesterol feedback regulation and NLRP3 inflammasome activation. According to the reports from P.L. Faust et al., ER stress usually regulates lipid metabolism through activation of SREBP-2, and does not depend on the concentration of intracellular cholesterol that we habitually believe [36]. Through in-depth analysis of our previous studies in vitro, we know that inhibition of ER stress has a significant inhibitory effect on AGEs induced cholesterol accumulation in HK-2 cells [10]. In addition, we have expanded the scope of our research, it can be found that there is a close relationship between ER stress and the activation of NLRP3 inflammasome under metabolic disorders [37]. ER stress inhibitors can suppress NLRP3 inflammasome activation in HepG2 and HK-2 cells [38, 39]. In this study, we found that EMPA can not only reduce the increase of GRP-78 and CHOP induced by AGEs in vivo, but also reduce the increase of GRP-78 and CHOP caused by AGEs in vitro, suggesting that EMPA can alleviate the lipid deposition and inflammatory response induced by AGEs through inhibiting ER stress. Since the AGEs-RAGE axis could activate ER stress and influence the progression of DKD, we think it is essential to evaluate the levels of AGEs and RAGE. Through the data obtained from the experiment, we know that the serum AGEs levels and renal RAGE protein levels were increased in $\mathrm{db} / \mathrm{db}$ mice compared with those in $\mathrm{db} / \mathrm{m}$ mice and were even higher in $\mathrm{db} / \mathrm{db}$ mice fed with a high-AGE diet. However, EMPA had a significant inhibitory effect on serum AGEs and renal RAGE protein levels in basal diet-fed $\mathrm{db} / \mathrm{db}$ mice and high-AGEs diet-fed db/db mice. Moreover, EMPA reduced the protein level of RAGE in AGEs-treated HK-2 cells. These results suggested that EMPA may reduce renal tubular lipid accumulation and inflammation by decreasing AGEs formation and inhibiting the AGEs-RAGE signaling pathway, thus suppressing the ER stress, followed by the inhibition of SCAP-SREBP-2- HMGCoAR/LDLr and the NLRP3 inflammasome pathway. In addition, EMPA showed the ability to decrease the levels of u-NGAL, SCR, BUN and 24-hour urine protein, suggesting that EMPA might improve renal function by reducing renal lipid deposition and the inflammatory response.

In summary, our study shows that AGEs-RAGE induces ER stress in renal tubular epithelial cells, which stimulates the expression of SCAP and SREBP-2, causing increased formation of SCAP-SREBP-2 complexes and enhanced transport of the complexes to the Golgi, where SREBP-2 is hydrolyzed. The active fragments of SREBP-2 (nSREBP-2) enter the nucleus, thereby upregulating HMGCoAR and LDLr expression levels and ultimately, increasing cholesterol synthesis and uptake. Simultaneously, the NLRP3 inflammasome is activated by AGEs and promotes the 
release of IL-1 $\beta$, leading to renal tubulointerstitial inflammation. However, EMPA attenuates AGEs synthesis and inhibits the AGEs-RAGE signaling pathway, thus suppressing ER stress and prohibiting the SCAP-SREBP-2-LDLr/HMACoAR and NLRP3 inflammasome pathways, thereby alleviating renal lipid accumulation and inflammation and finally preventing the development of DKD (Fig. 7).

\section{Declaration}

\section{Ethics approval and consent to participate}

Not applicable

\section{Consent for publication}

Not applicable.

\section{Availability of data and materials}

All data generated or analyzed during this study are included in this published article.

\section{Funding}

This work was supported by grants from National Natural Science Youth Foundation of China (grant no. 81700632 to Hong Sun; grant no. 81900794 to Zheng Liu), the Natural Science Youth Foundation of Jiangsu Province (grant no. BK20170366 to Hong Sun), People's Livelihood Science and Technology of Suzhou (grant no. SYS2020104 to Hong Sun), and China Postdoctoral Science Foundation (2020M671559 to Juan Chen).

\section{Authors' contributions}

HS designed the experiment. ZL, JC and LS performed experiments. JJ and HS revised the manuscript. All authors read and approved the final manuscript.

\section{Acknowledgments}

We would like to express our heartfelt gratitude to the Department of Endocrinology, The First Affiliated Hospital of Soochow University

\section{Authors' information}

${ }^{1}$ Department of Endocrinology and Metabolism, The First Affiliated Hospital of Soochow University, Suzhou, Jiangsu, China

${ }^{2}$ Department of Endocrinology, Jiangsu Province Hospital of Chinese Medicine, Affiliated Hospital of Nanjing University of Chinese Medicine, Nanjing, China

${ }^{3}$ Department of Nephrology, The First Affiliated Hospital of Soochow University, Suzhou, Jiangsu, China 


\section{References}

[1] R.Z. Alicic, M.T. Rooney, K.R. Tuttle, Diabetic Kidney Disease: Challenges, Progress, and Possibilities, Clinical journal of the American Society of Nephrology : CJASN 12 (2017) 2032-2045.

[2] A. Kumar Pasupulati, P.S. Chitra, G.B. Reddy, Advanced glycation end products mediated cellular and molecular events in the pathology of diabetic nephropathy, Biomolecular concepts 7 (2016) 293-309.

[3] R. Nishad, P. Meshram, A.K. Singh, G.B. Reddy, A.K. Pasupulati, Activation of Notch1 signaling in podocytes by glucose-derived AGEs contributes to proteinuria, BMJ open diabetes research \& care 8 (2020).

[4] A. Ojima, T. Matsui, Y. Nishino, N. Nakamura, S. Yamagishi, Empagliflozin, an Inhibitor of Sodium-Glucose Cotransporter 2 Exerts Anti-Inflammatory and Antifibrotic Effects on Experimental Diabetic Nephropathy Partly by Suppressing AGEs-Receptor Axis, Hormone and metabolic research $=$ Hormon- und Stoffwechselforschung $=$ Hormones et metabolisme 47 (2015) 686-692.

[5] R. Haraguchi, Y. Kohara, K. Matsubayashi, R. Kitazawa, S. Kitazawa, New Insights into the Pathogenesis of Diabetic Nephropathy: Proximal Renal Tubules Are Primary Target of Oxidative Stress in Diabetic Kidney, Acta histochemica et cytochemica 53 (2020) 21-31.

[6] A. Sotokawauchi, N. Nakamura, T. Matsui, Y. Higashimoto, S.I. Yamagishi, Glyceraldehyde-Derived Pyridinium Evokes Renal Tubular Cell Damage via RAGE Interaction, International journal of molecular sciences 21 (2020).

[7] K. Kaifu, S. Ueda, N. Nakamura, T. Matsui, N. Yamada-Obara, R. Ando, Y. Kaida, M. Nakata, M. Matsukuma-Toyonaga, Y. Higashimoto, K. Fukami, Y. Suzuki, S. Okuda, S.I. Yamagishi, Advanced glycation end products evoke inflammatory reactions in proximal tubular cells via autocrine production of dipeptidyl peptidase-4, Microvascular research 120 (2018) 90-93.

[8] Y. Ishibashi, S. Yamagishi, T. Matsui, K. Ohta, R. Tanoue, M. Takeuchi, S. Ueda, K. Nakamura, S. Okuda, Pravastatin inhibits advanced glycation end products (AGEs)-induced proximal tubular cell apoptosis and injury by reducing receptor for AGEs (RAGE) level, Metabolism: clinical and experimental 61 (2012) 1067-1072.

[9] Y. Yuan, H. Sun, Z. Sun, Advanced glycation end products (AGEs) increase renal lipid accumulation: a pathogenic factor of diabetic nephropathy (DN), Lipids in health and disease 16 (2017) 126.

[10] H. Sun, Y. Yuan, Z. Sun, Update on Mechanisms of Renal Tubule Injury Caused by Advanced Glycation End Products, BioMed research international 2016 (2016) 5475120.

[11] M.S. Brown, J.L. Goldstein, The SREBP pathway: regulation of cholesterol metabolism by proteolysis of a membrane-bound transcription factor, Cell 89 (1997) 331-340.

[12] J.L. Goldstein, R.A. DeBose-Boyd, M.S. Brown, Protein sensors for membrane sterols, Cell 124 (2006) 35-46.

[13] J. Sakai, R.B. Rawson, The sterol regulatory element-binding protein pathway: control of lipid homeostasis through regulated intracellular transport, Current opinion in lipidology 12 (2001) 261-266.

[14] H. Sun, Y. Yuan, Z.L. Sun, Cholesterol Contributes to Diabetic Nephropathy through SCAP-SREBP-2 Pathway, International journal of endocrinology 2013 (2013) 592576.

[15] J. Nespoux, V. Vallon, SGLT2 inhibition and kidney protection, Clin Sci (Lond) 132 (2018) 
1329-1339.

[16] D. Margonato, G. Galati, S. Mazzetti, R. Cannistraci, G. Perseghin, A. Margonato, A. Mortara, Renal protection: a leading mechanism for cardiovascular benefit in patients treated with SGLT2 inhibitors, Heart failure reviews (2020).

[17] J. Hodrea, D.B. Balogh, A. Hosszu, L. Lenart, B. Besztercei, S. Koszegi, N. Sparding, F. Genovese, L.J. Wagner, A.J. Szabo, A. Fekete, Reduced O-GlcNAcylation and tubular hypoxia contribute to the antifibrotic effect of SGLT2 inhibitor dapagliflozin in the diabetic kidney, American journal of physiology. Renal physiology 318 (2020) F1017-F1029.

[18] T.C. Woods, R. Satou, K. Miyata, A. Katsurada, C.M. Dugas, N.C. Klingenberg, V.A. Fonseca, L.G. Navar, Canagliflozin Prevents Intrarenal Angiotensinogen Augmentation and Mitigates Kidney Injury and Hypertension in Mouse Model of Type 2 Diabetes Mellitus, American journal of nephrology 49 (2019) 331-342.

[19] H. Yaribeygi, N. Katsiki, A.E. Butler, A. Sahebkar, Effects of antidiabetic drugs on NLRP3 inflammasome activity, with a focus on diabetic kidneys, Drug discovery today 24 (2019) 256-262.

[20] X.X. Wang, J. Levi, Y. Luo, K. Myakala, M. Herman-Edelstein, L. Qiu, D. Wang, Y. Peng, A. Grenz, S. Lucia, E. Dobrinskikh, V.D. D'Agati, H. Koepsell, J.B. Kopp, A.Z. Rosenberg, M. Levi, SGLT2 Protein Expression Is Increased in Human Diabetic Nephropathy: SGLT2 PROTEIN INHIBITION DECREASES RENAL LIPID ACCUMULATION, INFLAMMATION, AND THE DEVELOPMENT OF NEPHROPATHY IN DIABETIC MICE, The Journal of biological chemistry 292 (2017) 5335-5348.

[21] B. Sowndhar Rajan, S. Manivasagam, S. Dhanusu, N. Chandrasekar, K. Krishna, L.P. Kalaiarasu, A.A. Babu, E. Vellaichamy, Diet with high content of advanced glycation end products induces systemic inflammation and weight gain in experimental mice: Protective role of curcumin and gallic acid, Food and chemical toxicology : an international journal published for the British Industrial Biological Research Association 114 (2018) 237-245.

[22] H. Sun, Z. Sun, Z. Varghese, Y. Guo, J.F. Moorhead, R.J. Unwin, X.Z. Ruan, Nonesterified free fatty acids enhance the inflammatory response in renal tubules by inducing extracellular ATP release, American journal of physiology. Renal physiology 319 (2020) F292-F303.

[23] Y.Y. Qiu, L.Q. Tang, Roles of the NLRP3 inflammasome in the pathogenesis of diabetic nephropathy, Pharmacological research : the official journal of the Italian Pharmacological Society 114 (2016) 251-264.

[24] K.H. Huang, S.S. Guan, W.H. Lin, C.T. Wu, M.L. Sheu, C.K. Chiang, S.H. Liu, Role of Calbindin-D28k in Diabetes-Associated Advanced Glycation End-Products-Induced Renal Proximal Tubule Cell Injury, Cells 8 (2019).

[25] N. Pathomthongtaweechai, S. Chutipongtanate, AGE/RAGE signaling-mediated endoplasmic reticulum stress and future prospects in non-coding RNA therapeutics for diabetic nephropathy, Biomedicine \& pharmacotherapy = Biomedecine \& pharmacotherapie 131 (2020) 110655.

[26] Z. Wang, T. Jiang, J. Li, G. Proctor, J.L. McManaman, S. Lucia, S. Chua, M. Levi, Regulation of renal lipid metabolism, lipid accumulation, and glomerulosclerosis in FVBdb/db mice with type 2 diabetes, Diabetes 54 (2005) 2328-2335.

[27] M. Herman-Edelstein, P. Scherzer, A. Tobar, M. Levi, U. Gafter, Altered renal lipid metabolism and renal lipid accumulation in human diabetic nephropathy, Journal of lipid research 55 (2014) 561-572. 
[28] Y. Zhang, K.L. Ma, J. Liu, Y. Wu, Z.B. Hu, L. Liu, J. Lu, X.L. Zhang, B.C. Liu, Inflammatory stress exacerbates lipid accumulation and podocyte injuries in diabetic nephropathy, Acta diabetologica 52 (2015) 1045-1056.

[29] E.S. Lee, M.H. Kwon, H.M. Kim, N. Kim, Y.M. Kim, H.S. Kim, E.Y. Lee, C.H. Chung, Dibenzoylmethane ameliorates lipid-induced inflammation and oxidative injury in diabetic nephropathy, The Journal of endocrinology 240 (2019) 169-179.

[30] S.C.W. Tang, W.H. Yiu, Innate immunity in diabetic kidney disease, Nature reviews. Nephrology 16 (2020) 206-222.

[31] S. Son, I. Hwang, S.H. Han, J.S. Shin, O.S. Shin, J.W. Yu, Advanced glycation end products impair NLRP3 inflammasome-mediated innate immune responses in macrophages, The Journal of biological chemistry 292 (2017) 20437-20448.

[32] J. Hong, G. Li, Q. Zhang, J. Ritter, W. Li, P.L. Li, D-Ribose Induces Podocyte NLRP3 Inflammasome Activation and Glomerular Injury via AGEs/RAGE Pathway, Frontiers in cell and developmental biology 7 (2019) 259.

[33] Y. Song, Y. Wang, Y. Zhang, W. Geng, W. Liu, Y. Gao, S. Li, K. Wang, X. Wu, L. Kang, C. Yang, Advanced glycation end products regulate anabolic and catabolic activities via NLRP3-inflammasome activation in human nucleus pulposus cells, Journal of cellular and molecular medicine 21 (2017) 1373-1387.

[34] C. Guo, Z. Chi, D. Jiang, T. Xu, W. Yu, Z. Wang, S. Chen, L. Zhang, Q. Liu, X. Guo, X. Zhang, W. Li, L. Lu, Y. Wu, B.L. Song, D. Wang, Cholesterol Homeostatic Regulator SCAP-SREBP2 Integrates NLRP3 Inflammasome Activation and Cholesterol Biosynthetic Signaling in Macrophages, Immunity 49 (2018) 842-856 e847.

[35] E. Benetti, R. Mastrocola, G. Vitarelli, J.C. Cutrin, D. Nigro, F. Chiazza, E. Mayoux, M. Collino, R. Fantozzi, Empagliflozin Protects against Diet-Induced NLRP-3 Inflammasome Activation and Lipid Accumulation, The Journal of pharmacology and experimental therapeutics 359 (2016) 45-53.

[36] P.L. Faust, W.J. Kovacs, Cholesterol biosynthesis and ER stress in peroxisome deficiency, Biochimie 98 (2014) 75-85.

[37] T. Ji, Y. Han, W. Yang, B. Xu, M. Sun, S. Jiang, Y. Yu, Z. Jin, Z. Ma, Y. Yang, W. Hu, Endoplasmic reticulum stress and NLRP3 inflammasome: Crosstalk in cardiovascular and metabolic disorders, Journal of cellular physiology (2019).

[38] N. Bo, H. Yilin, Y. Chaoyue, L. Lu, Y. Yuan, Acrylamide induces NLRP3 inflammasome activation via oxidative stress- and endoplasmic reticulum stress-mediated MAPK pathway in HepG2 cells, Food and chemical toxicology : an international journal published for the British Industrial Biological Research Association 145 (2020) 111679.

[39] J. Wang, Y. Wen, L.L. Lv, H. Liu, R.N. Tang, K.L. Ma, B.C. Liu, Involvement of endoplasmic reticulum stress in angiotensin II-induced NLRP3 inflammasome activation in human renal proximal tubular cells in vitro, Acta pharmacologica Sinica 36 (2015) 821-830. 
661

662

\section{Figure legends}

Fig. 1 Effects of EMPA on biochemical characteristics of diabetic mice. The levels of FBG (A), AGEs (B), TG (C), TC (D), SCR (E), BUN (F), u-NGAL (G) and 24-hour urine protein $(\mathrm{H})$ in mice. The values are expressed as the mean \pm S.E.M. of 5 independent experiments. ${ }^{*} P<0.05$ and ${ }^{*} * P<0.01$ versus the $\mathrm{db} / \mathrm{m}$ group; ${ }^{\#} P<0.05$ and ${ }^{\# \#} P<0.01$ versus the $\mathrm{db} / \mathrm{db}$ group; ${ }^{\boldsymbol{\Delta}} P<0.05$ and ${ }^{\boldsymbol{\Delta}} P<0.01$ versus the AGEs+db/db group; ns = not significant.

Fig. 2 Effects of EMPA on renal lipid accumulation in vivo and in vitro. Renal Oil Red $\mathrm{O}$ staining and the semi-quantitative analysis for the percent of the positive areas in each group (A). The intracellular cholesterol contents in the kidneys of mice (B). Oil Red O staining in the HK-2 cells and the positive percentage of HK-2 cells (C). The intracellular cholesterol content in HK-2 cells (D). Oil Red O staining was observed under a light microscope $(400 \times)$. The percentage of positive staining areas in the kidneys of mice was semi-quantitative analyzed by Image J. The positive percentage of HK-2 cells was counted from 5 experiments. Values of intracellular cholesterol content are expressed as the means \pm S.E.M. of 5 independent experiments. $* * P<0.01$ versus the $\mathrm{db} / \mathrm{m}$ group or the control cells $(\mathrm{Ctr}) ;{ }^{\#} P<0.05$ and ${ }^{\# \#} P<0.01$ versus $\mathrm{db} / \mathrm{db}$ group or AGEs-BSA treated cells; ${ }^{\boldsymbol{\Lambda}} P<0.05$ and ${ }^{\boldsymbol{\Delta}}{ }^{\boldsymbol{}} P<0.01$ versus the AGEs+db/db group.

Fig. 3 Effects of EMPA on HMGCoAR, LDLr, SREBP-2, nSREBP-2 and SCAP expression in the kidneys of diabetic mice and HK-2 cells. The protein expression of HMGCoAR, LDLr, SREBP-2, nSREBP-2 and SCAP in the kidneys of mice (A) and in HK-2 cells (B) was determined by Western blotting. ImageJ was used to quantify the relative levels of proteins. GAPDH or Lamin A was used as an internal control. The values are expressed as the mean \pm S.E.M. of 5 independent experiments. $* P<0.05$ and ${ }^{*} P<<0.01$ versus the $\mathrm{db} / \mathrm{m}$ group or the control cells $(\mathrm{Ctr}) ;{ }^{\#} P<0.05$ and ${ }^{\# \#} P<$ 0.01 , versus $\mathrm{db} / \mathrm{db}$ group or AGEs-BSA treated cells; ${ }^{\boldsymbol{\Delta}} P<0.01$ versus the AGEs+db/db group.

Fig. 4 Effect of EMPA on SCAP-SREBP-2 complexes in HK-2 cells. The translocation of GFP-SCAP from the ER to the Golgi was investigated using confocal microscopy after staining with anti-Golgi antibody. EMPA inhibited the translocation of GFP-SCAP from the ER to the Golgi in HK-2 cells (A). The interaction between SCAP and SREBP-2 proteins was investigated using Co-IP. AGEs-BSA increased the interactions of SCAP and SREBP-2 in HK-2 cells, whereas this could be inhibited by $\operatorname{EMPA}(\mathbf{B})$

Fig. 5 Effects of EMPA on renal tubular inflammation in diabetic mice and HK-2 cells. HE (A) staining in the kidneys of mice was observed under a light microscope $(\times 400)$. The protein expression of NLRP3 and IL-1 $\beta$ in the kidneys of mice $(\mathbf{B})$ and in HK-2 cells $(\mathbf{C})$ was determined by Western blotting. ImageJ was used to quantify the relative levels of proteins. GAPDH was used as an internal control. Values are expressed as the mean \pm S.E.M. of 5independent experiments. NLRP3 inflammasome activation was 
detected by coimmunoprecipitation $(\mathbf{D}) . * * P<0.01$ versus the $\mathrm{db} / \mathrm{m}$ group or the control cells $(\mathrm{Ctr}) ;{ }^{\#} P<0.05$ and ${ }^{\# \#} P<0.01$ versus db/db group or AGEs-BSA treated cells; ${ }^{\wedge} P<0.05$ versus the AGEs $+\mathrm{db} / \mathrm{db}$ group.

Fig. 6 Effects of EMPA on ER stress and RAGE expression. The protein expression of GRP78, CHOP and RAGE in the kidneys of mice (A) and HK-2 cells (B) was determined by Western blotting. ImageJ was used to quantify the relative levels of proteins. GAPDH was used as an internal control. The values are expressed as the mean \pm S.E.M. of 5 independent experiments. $* * P<0.01$ versus the $\mathrm{db} / \mathrm{m}$ group or the control cells $(\mathrm{Ctr}) ;{ }^{\#} P<0.05$ and ${ }^{\# \#} P<0.01$ versus db/db group or AGEs-BSA treated cells; ${ }^{\wedge} P<0.05$ and ${ }^{\wedge}{ }^{\wedge} P<0.01$ versus the AGEs+db/db group.

Fig. 7 Role of EMPA in AGEs induced renal tubular lipid accumulation. AGEs-RAGE induces ER stress in the renal tubular epithelial cells, which stimulates the expression of SCAP and SREBP-2, causing increased formation of SCAP-SREBP-2 complexes and enhanced transport of the complexes to the Golgi, where SREBP-2 is hydrolyzed. The active fragments of SREBP-2 (nSREBP-2) enter the nucleus, thereby upregulating HMGCoAR and LDLr expression levels and ultimately, increase the cholesterol synthesis and uptake. Simultaneously, the NLRP3 inflammasome is activated and promotes the release of IL-1 $\beta$, leading to renal tubulointerstitial inflammation. However, EMPA attenuates AGEs synthesis and inhibits the AGEs-RAGE signaling pathway, thus suppressing ER stress and prohibiting the SCAP-SREBP-2-LDLr/HMACoAR and NLRP3 inflammasome pathways, thereby alleviating renal lipid accumulation and inflammation. 

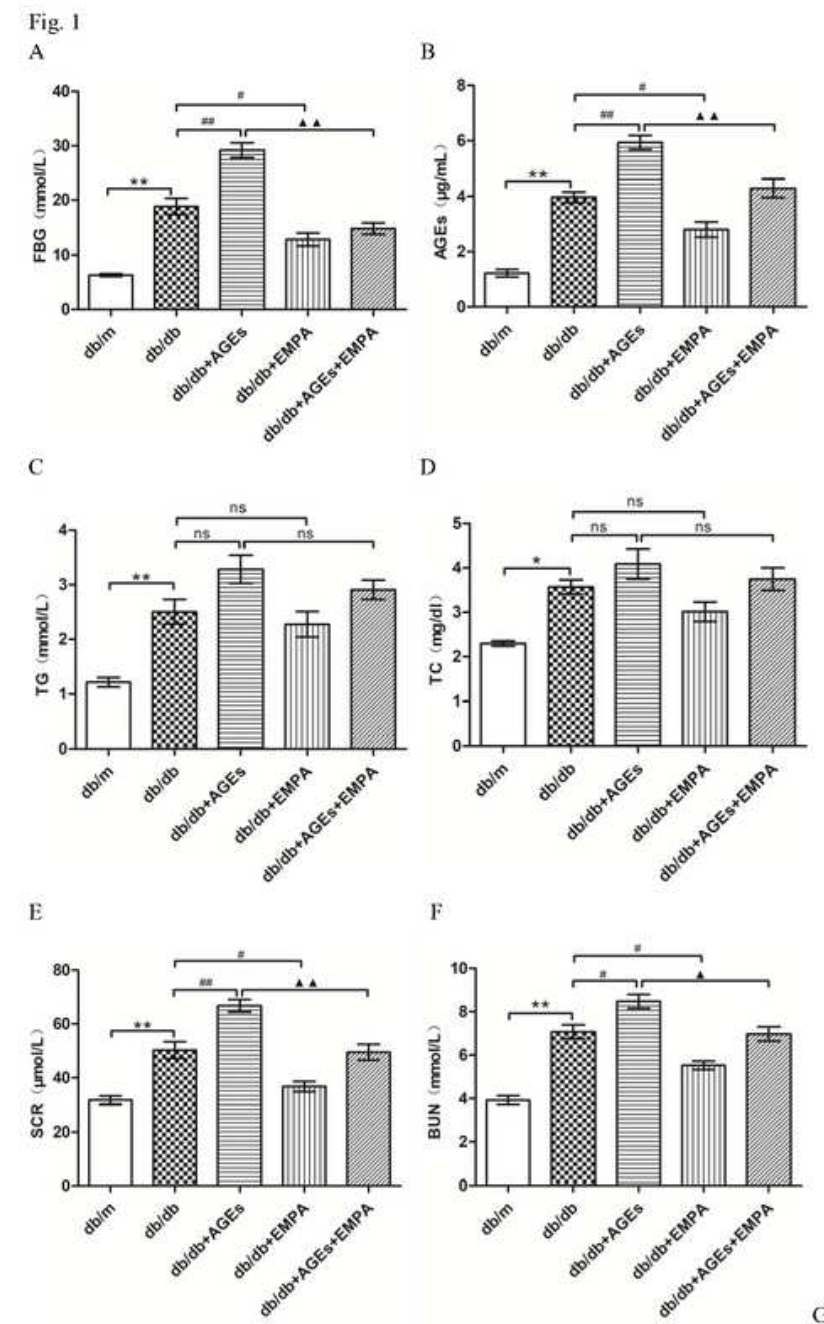

F
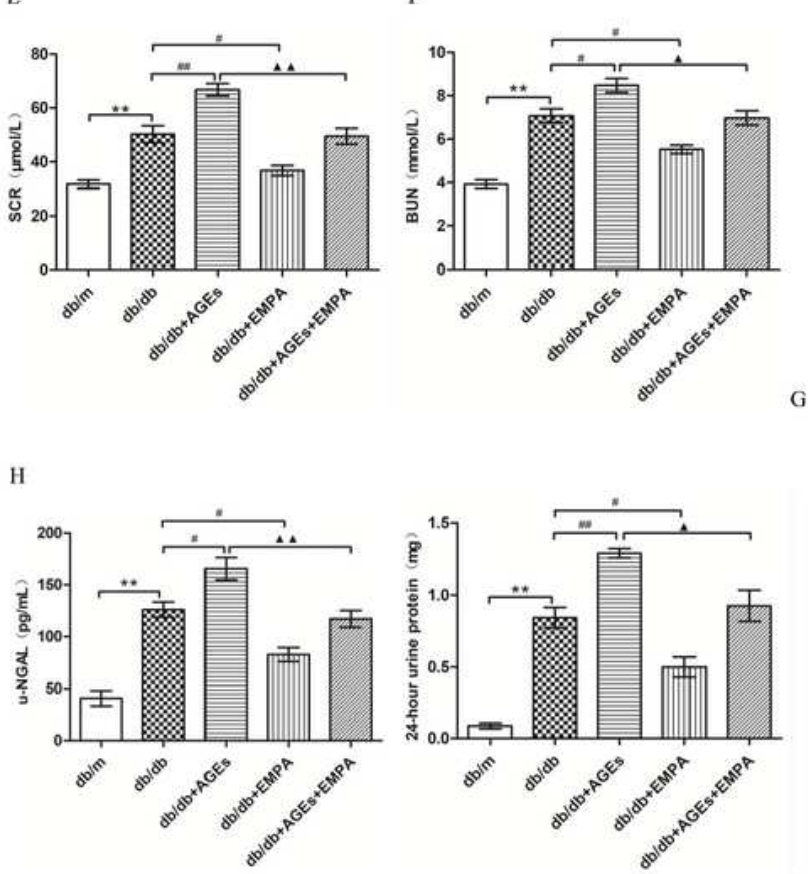

Figure 1

Effects of EMPA on biochemical characteristics of diabetic mice. The levels of FBG (A), AGEs (B), TG (C), TC (D), SCR (E), BUN (F), u-NGAL (G) and 24-hour urine protein $(H)$ in mice. The values are expressed as the mean \pm S.E.M. of 5 independent experiments. ${ }^{*} \mathrm{P}<0.05$ and ${ }^{*} \mathrm{P}<0.01$ versus the $\mathrm{db} / \mathrm{m}$ group; \# $\mathrm{P}<$ 


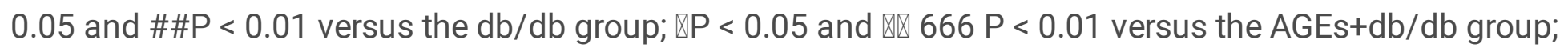
ns $=$ not significant.
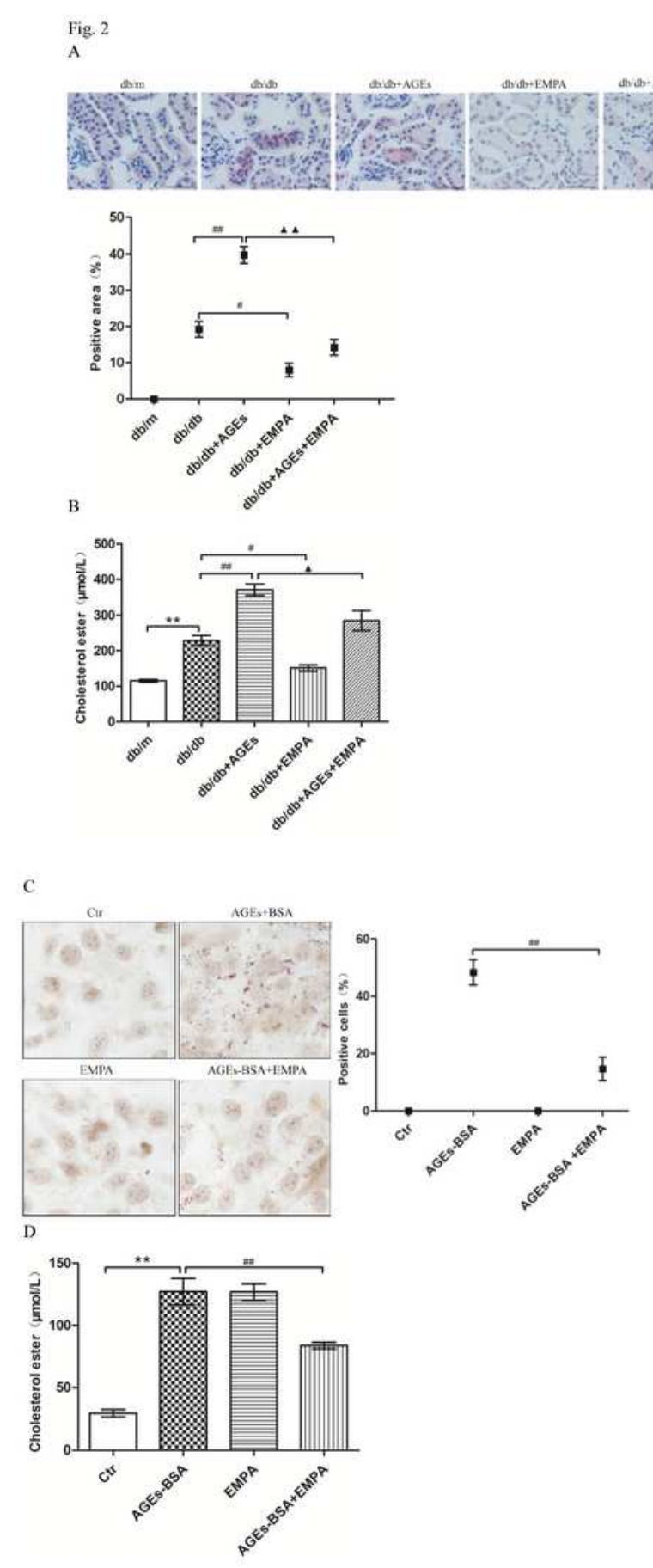

\section{Figure 2}

Effects of EMPA on renal lipid accumulation in vivo and in vitro. Renal Oil Red $O$ staining and the semiquantitative analysis for the percent of the positive areas in each group (A). The intracellular cholesterol contents in the kidneys of mice (B). Oil Red O staining in the HK-2 cells and the positive percentage of HK- 
2 cells (C). The intracellular cholesterol content in HK-2 cells (D). Oil Red O staining was observed under a light microscope (400x). The percentage of positive staining areas in the kidneys of mice was semiquantitative analyzed by Image $\mathrm{J}$. The positive percentage of HK-2 cells was counted from 5 experiments. Values of intracellular cholesterol content are expressed as the means $\pm S . E . M$. of 5 independent experiments. ${ }^{\star *} P<0.01$ versus the $\mathrm{db} / \mathrm{m}$ group or the control cells (Ctr); $\mathrm{P}<0.05$ and \#\# $\mathrm{P}<0.01$ versus

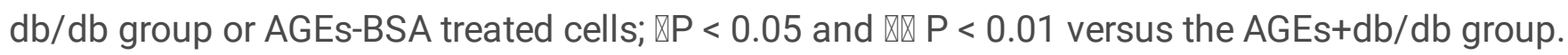
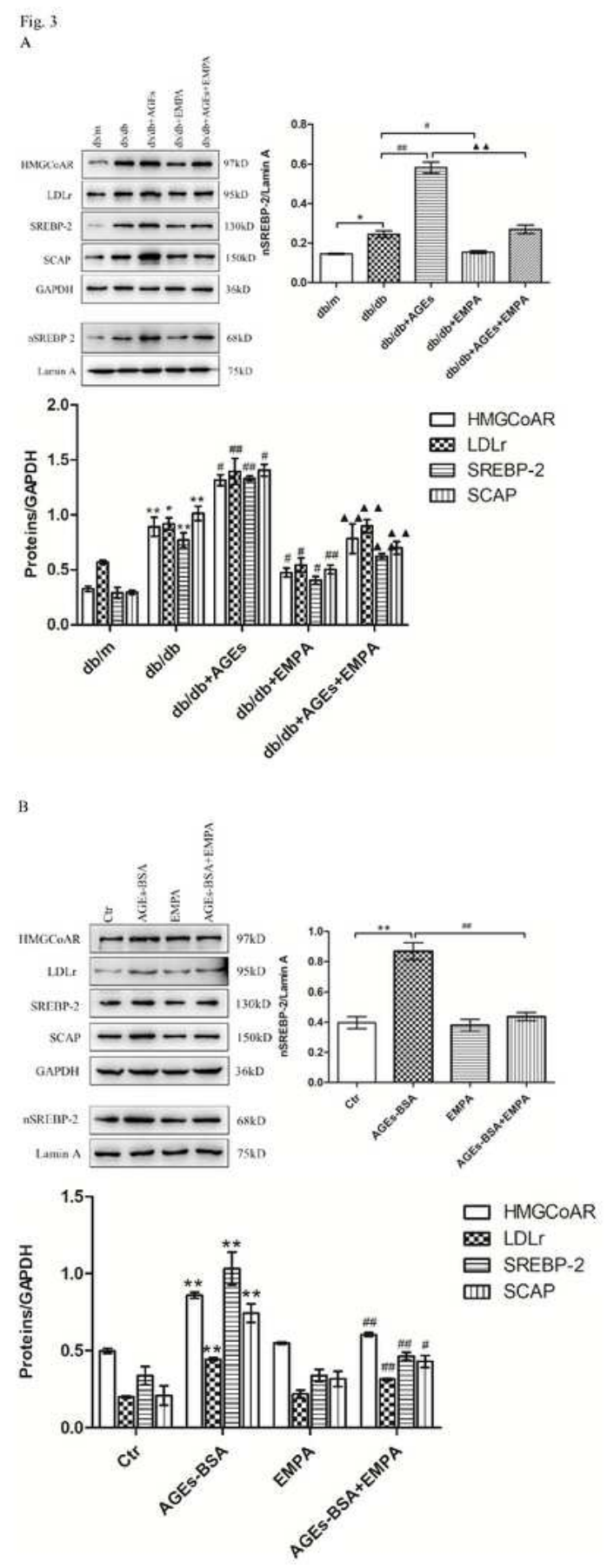

Figure 3 
Effects of EMPA on HMGCoAR, LDLr, SREBP-2, nSREBP-2 and SCAP expression in the kidneys of diabetic mice and HK-2 cells. The protein expression of HMGCoAR, LDLr, SREBP-2, nSREBP-2 and SCAP in the kidneys of mice (A) and in HK-2 cells (B) was determined by Western blotting. ImageJ was used to quantify the relative levels of proteins. GAPDH or Lamin A was used as an internal control. The values are expressed as the mean \pm S.E.M. of 5 independent experiments. ${ }^{*} \mathrm{P}<0.05$ and ${ }^{*} \mathrm{P}<0.01$ versus the $\mathrm{db} / \mathrm{m}$ group or the control cells (Ctr); \# P $<0.05$ and \#\# $\mathrm{P}<0.01$, versus $\mathrm{db} / \mathrm{db}$ group or AGEs-BSA treated cells; $\mathbb{Q} \mathrm{P}<0.01$ versus the $A G E s+d b / d b$ group.

Fig. 4

A

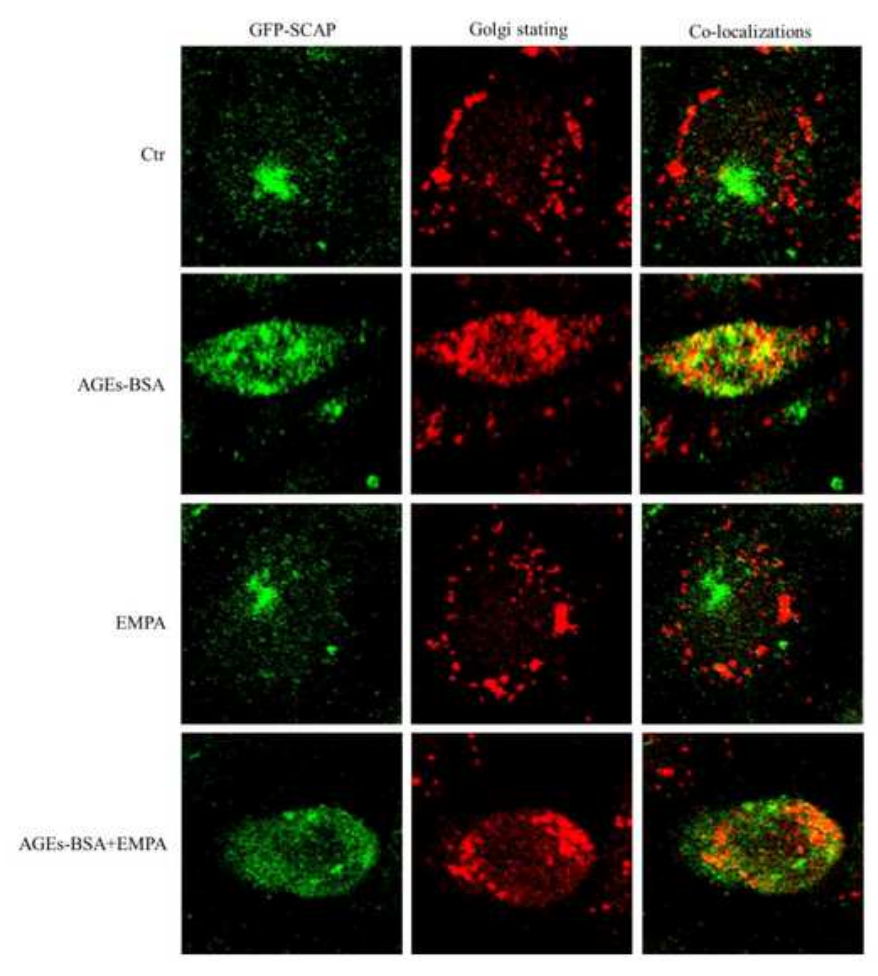

B

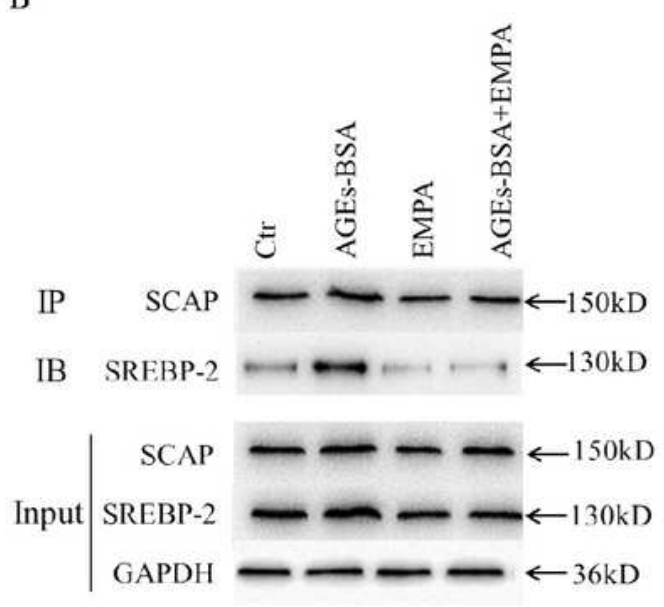


Figure 4

Effect of EMPA on SCAP-SREBP-2 complexes in HK-2 cells. The translocation of GFP-SCAP from the ER to the Golgi was investigated using confocal microscopy after staining with anti-Golgi antibody. EMPA inhibited the translocation of GFP-SCAP from the ER to the Golgi in HK-2 cells (A). The interaction between SCAP and SREBP-2 proteins was investigated using Co-IP. AGEs-BSA increased the interactions of SCAP and SREBP-2 in HK-2 cells, whereas this could be inhibited by EMPA (B)

Fig. 5

A

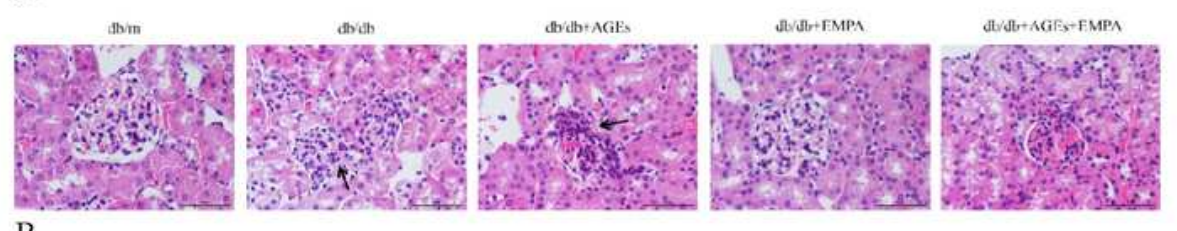

B

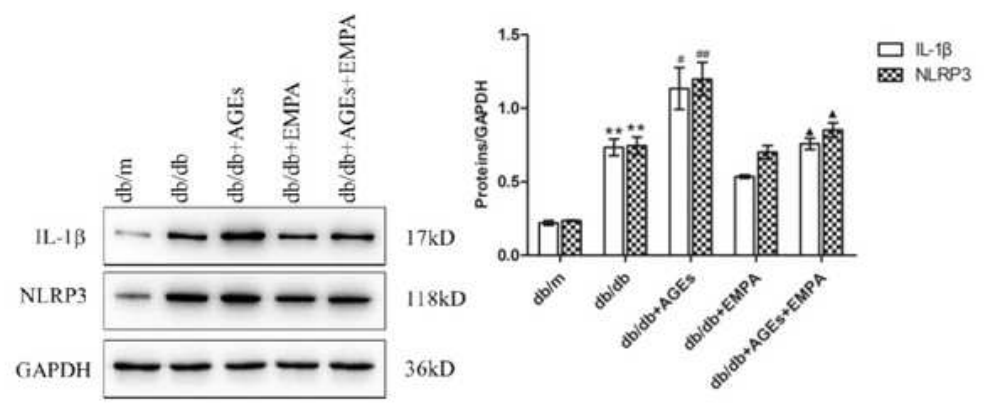

C

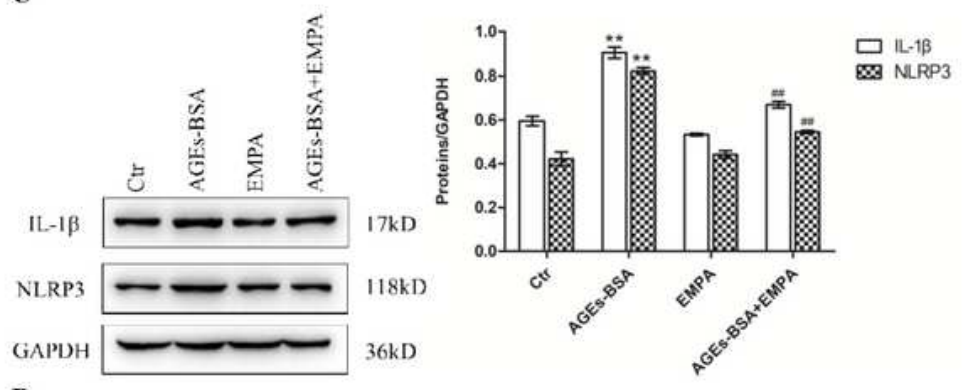

$\mathrm{D}$

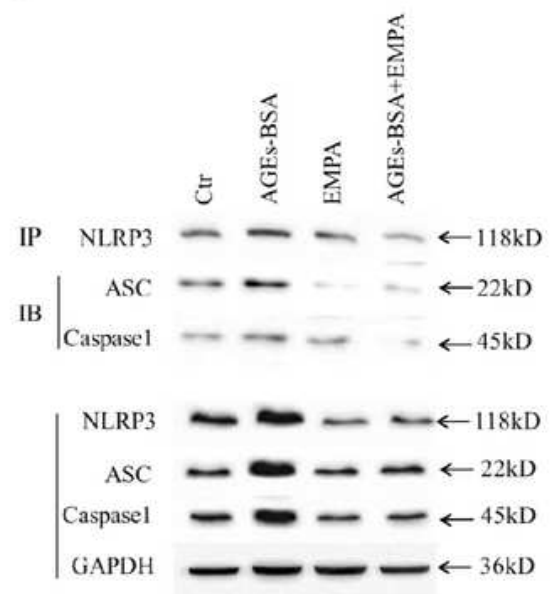




\section{Figure 5}

Effects of EMPA on renal tubular inflammation in diabetic mice and HK-2 cells. HE (A) staining in the kidneys of mice was observed under a light microscope $(\times 400)$. The protein expression of NLRP3 and IL$1 \beta$ in the kidneys of mice (B) and in HK-2 cells (C) was determined by Western blotting. ImageJ was used to quantify the relative levels of proteins. GAPDH was used as an internal control. Values are expressed as the mean \pm S.E.M. of 5independent experiments. NLRP3 inflammasome activation was detected by coimmunoprecipitation (D). ${ }^{*} P<0.01$ versus the $\mathrm{db} / \mathrm{m}$ group or the control cells (Ctr); \# $\mathrm{P}<0.05$ and \#\# $P<0.01$ versus $d b / d b$ group or AGEs-BSA treated cells; $\otimes P<0.05$ versus the AGEs $+d b / d b$ group.

Fig. 6

A

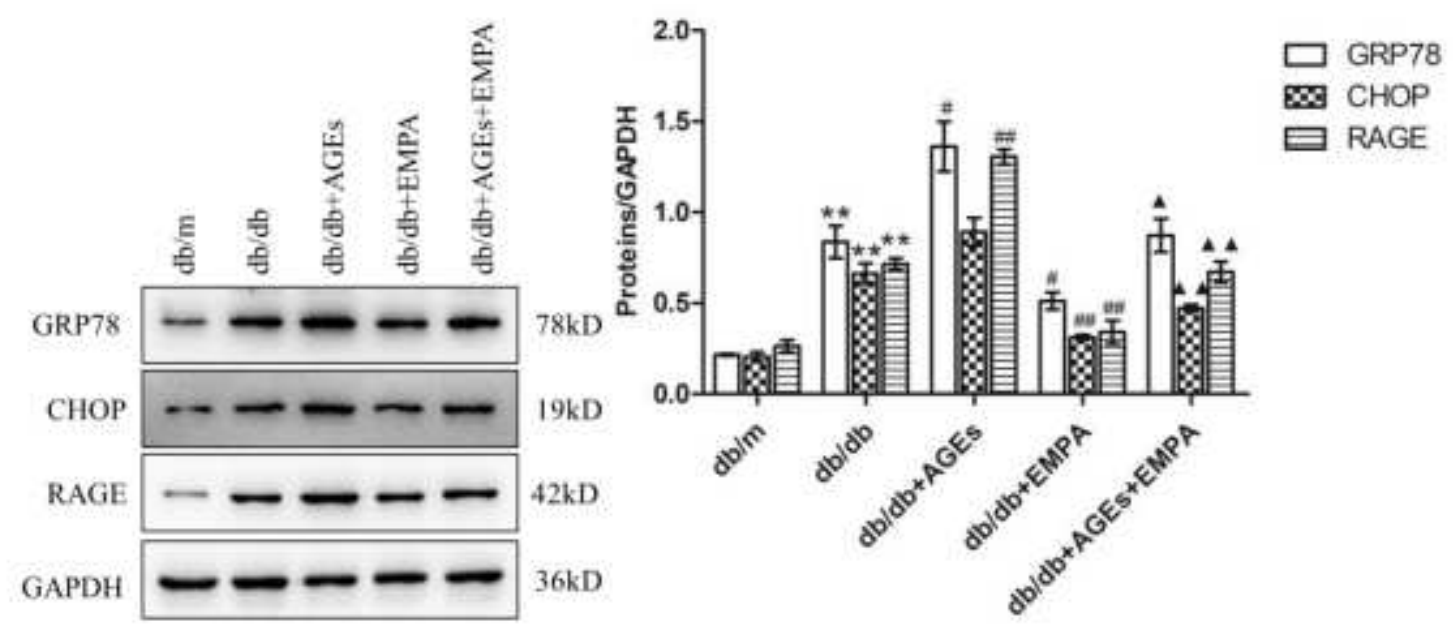

B
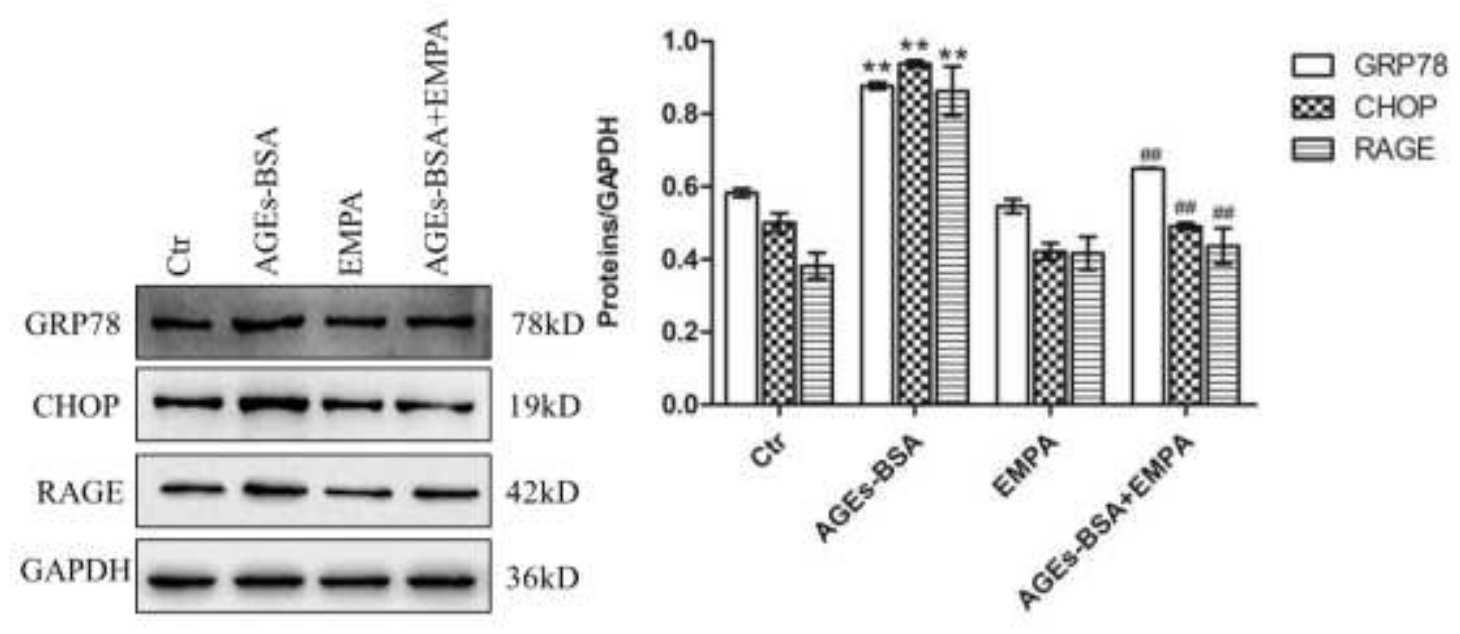

Figure 6

Effects of EMPA on ER stress and RAGE expression. The protein expression of GRP78, CHOP and RAGE in the kidneys of mice (A) and HK-2 cells (B) was determined by Western blotting. ImageJ was used to quantify the relative levels of proteins. GAPDH was used as an internal control. The values are expressed as the mean \pm S.E.M. of 5 independent experiments. ${ }^{*} \mathrm{P}<0.01$ versus the $\mathrm{db} / \mathrm{m}$ group or the control cells 


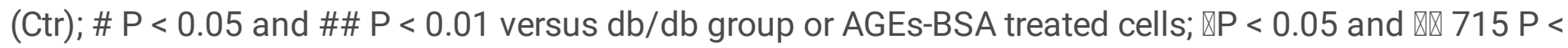
0.01 versus the $A G E s+d b / d b$ group.

Fig. 7

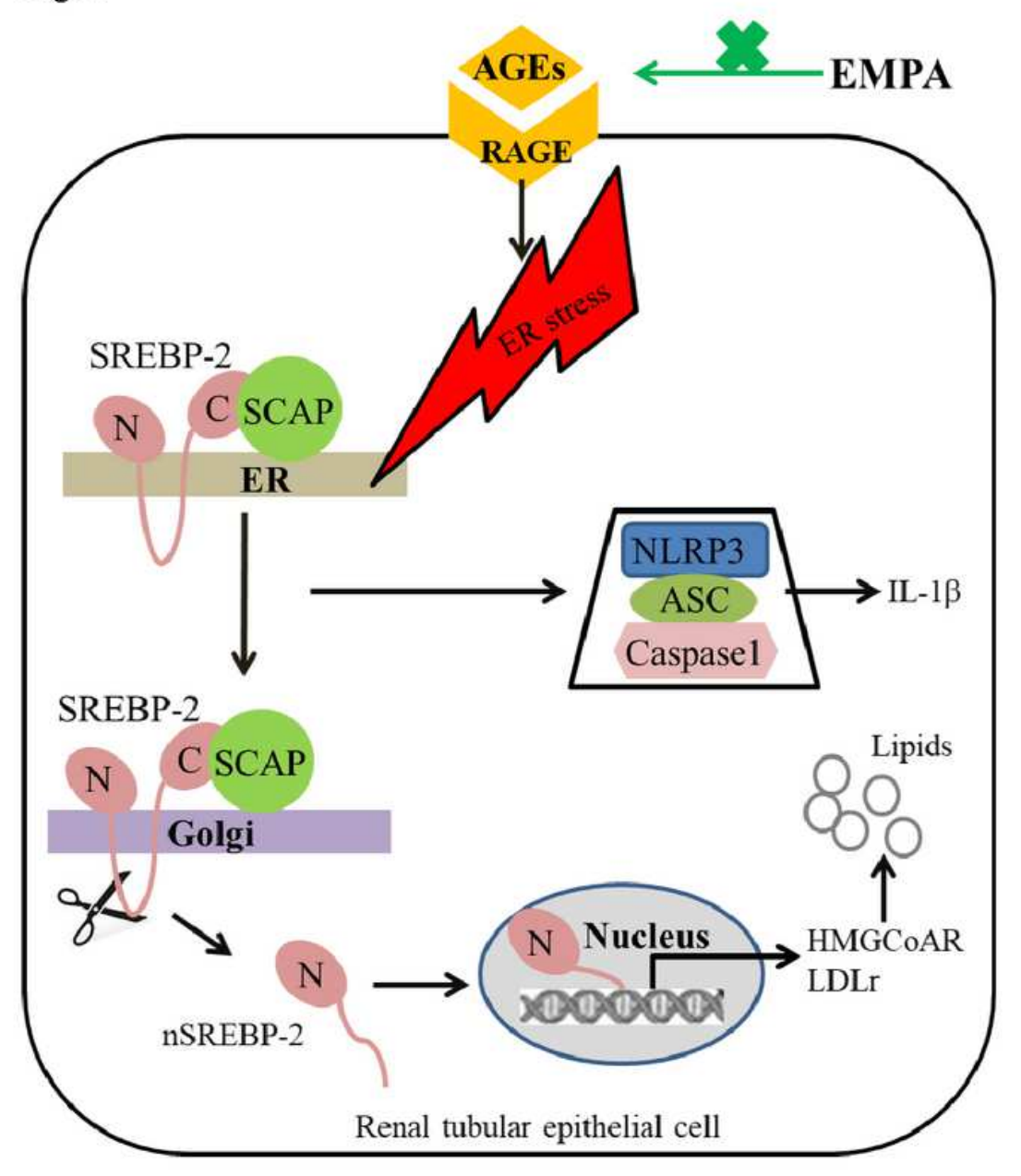

\section{Figure 7}

Role of EMPA in AGEs induced renal tubular lipid accumulation. AGEs-RAGE induces ER stress in the renal tubular epithelial cells, which stimulates the expression of SCAP and SREBP-2, causing increased formation of SCAP-SREBP-2 complexes and enhanced transport of the complexes to the Golgi, where SREBP-2 is hydrolyzed. The active fragments of SREBP-2 (nSREBP-2) enter the nucleus, thereby upregulating HMGCOAR and LDLr expression levels and ultimately, increase the cholesterol synthesis and uptake. Simultaneously, the NLRP3 inflammasome is activated and promotes the release of IL-1 to renal tubulointerstitial inflammation. However, EMPA attenuates AGEs synthesis and inhibits the AGEs- 
RAGE signaling pathway, thus suppressing ER stress and prohibiting the SCAP-SREBP-2-LDLr/HMACoAR and NLRP3 inflammasome pathways, thereby alleviating renal lipid accumulation and inflammation. 